\title{
Molecular cloning, characterization and differential expression of DRK1 in Sporothrix schenckii
}

\author{
BINBIN HOU $^{1 *}$, ZHENYING ZHANG $^{1 *}$, FANGLIANG ZHENG $^{2}$ and XIAOMING LIU ${ }^{1}$ \\ ${ }^{1}$ Department of Dermatology, The First Affiliated Hospital of Dalian Medical University, Dalian, \\ Liaoning 116011; ${ }^{2}$ Key Laboratory of Animal Resource and Epidemic Disease Prevention, \\ Life Science School of Liaoning University, Shenyang, Liaoning 110036, P.R. China
}

Received September 5, 2012; Accepted November 2, 2012

DOI: $10.3892 /$ ijmm.2012.1193

\begin{abstract}
The dimorphism of Sporothrix schenckii (S. schenckii) reflects a developmental switch in morphology and lifestyle that is necessary for virulence. DRK1, a hybrid histidine kinase, functions as a global regulator of dimorphism and virulence in Blastomyces dermatitidis (B. dermatitidis) and Histoplasma capsulatum (H. capsulatum). The partial cDNA sequence of DRK1 of S. schenckii, designated SsDRK1, was obtained using degenerate primers based on the conserved domain of the DRK1 of other fungi. The complete cDNA sequence of SsDRK1 was obtained by 5' and 3' RACE. The full-length cDNA is $4743 \mathrm{bp}$ in size and has an open reading frame (ORF) of $4071 \mathrm{bp}$, encoding 1356 amino acid residues. The predicted molecular mass of $S s D R K 1$ is $147.3 \mathrm{kDa}$ with an estimated theoretical isoelectric point of 5.46. The deduced amino acid sequence of $S S D R K 1$ shows $65 \%$ identity to that of $B$. dermatitidis. The $S s D R K 1$ was predicted to be a soluble histidine kinase and to contain three parts: sensor domain, linker domain and functional domain. Quantitative real-time RT-PCR revealed that $S s D R K 1$ was more highly expressed in the yeast stage compared with that in the mycelial stage, which indicated that the SsDRK1 may be involved in the dimorphic switch in S. schenckii.
\end{abstract}

\section{Introduction}

The dimorphic fungus Sporothrix schenckii (S. schenckii) is the etiological agent of sporotrichosis, an important cutaneous

Correspondence to: Professor Xiaoming Liu and Dr Zhenying Zhang, Department of Dermatology, The First Affiliated Hospital of Dalian Medical University, 222 Zhongshan Road, Dalian, Liaoning 116011, P.R. China

E-mail: liuxiaoming1956@yahoo.cn

E-mail: zhangzhenying7983@yahoo.com.cn

*Contributed equally

Key words: DRK1, Sporothrix schenckii, gene cloning, differential expression mycosis with a worldwide distribution (1). S. schenckii grows at room temperature $\left(25^{\circ} \mathrm{C}\right)$ as a mold phase, while in vitro incubation of mold cultures at body temperature $\left(37^{\circ} \mathrm{C}\right)$ results in the production of yeast cells (2). These in vitro forms are virtually identical to the yeast cells of $S$. schenckii found in diseased tissue. Therefore, the formation of yeast cells was thought to be a requisite for the pathogenicity of $S$. schenckii. The mechanisms that regulate the dimorphic switch, however, remain unclear.

The mitogen-activated protein kinase (MAPK) cascade and cyclic AMP (cAMP) signaling pathways are known to be involved in fungal morphogenesis and pathogenic development. However, the MAPK and cAMP pathways are both activated by an upstream branch, two-component histidine kinase phospho-relay system. Nemecek et al (3) recently uncovered a long-sought regulator that controls the switch from a non-pathogenic mold form to a pathogenic yeast form in dimorphic fungi. They found that DRK1, a hybrid dimorphism-regulating histidine kinase, functions as a global regulator of dimorphism and virulence in Blastomyces dermatitidis (B. dermatitidis) and Histoplasma capsulatum (H. capsulatum). DRK1 is required for phase transition from mold to yeast, expression of virulence genes, and pathogenicity in vivo. Disruption of DRK1 locks $B$. dermatitidis in the mold form at temperatures $\left(37^{\circ} \mathrm{C}\right)$ that normally trigger phase transition to yeast. RNA silencing of DRK1 expression in $B$. dermatitidis results in impaired BAD1 expression, severe alterations in the cell wall, and reduction in transcription of $\alpha-(1,3)$-glucan synthase and the yeast-phase specific gene BYS1. In H. capsulatum, DRK1 also regulates expression of the yeast-phase specific genes CBP1, AGS1 and yps-3. We previously reported differentially expressed genes between the mycelial and the yeast phases of $S$. schenckii using 2DE. The expressed sequence tag of spotC homologous to the DRK1 histidine kinase from $B$. dermatitidis clearly increases in the yeast form of $S$. schenckii (4).

We describe the molecular cloning of the DRK1 gene from the yeast-form $S$. schenckii, designated $S s D R K 1$. We performed necessary function analysis of the $S s D R K 1$ gene as well as detection of the differential gene expression in the dimorphic switch of S. schenckii. These findings establish the primary foundation of understanding the function of SsDRK1. The cloning and characterization of the DRK1 gene in S. schenckii is reported for the first time. 


\section{Materials and methods}

Fungal strain, media and growth conditions. The strain of S. schenckii used, ATCC10268, was maintained at the Research Center for Pathogenic Fungi, Dalian Medical University, China. To obtain a mycelial culture, the ATCC10268 isolate was inoculated on Sabouraud dextrose agar (SDA) medium and incubated at $25^{\circ} \mathrm{C}$. The mycelial colonies thus obtained were inoculated in Sabouraud's fluid medium and cultured with shaking at $100 \mathrm{rpm}$ at $25^{\circ} \mathrm{C}$ for $72 \mathrm{~h}$. To achieve the switch of S. schenckii from the mycelial to the yeast phase, mycelial colonies were transferred to brain heart infusion (BHI) liquid medium at $37^{\circ} \mathrm{C}$ and shaken at $100 \mathrm{rpm}$ for $96 \mathrm{~h}$. Mycelial and yeast pellets were collected by centrifugation and stored at $-80^{\circ} \mathrm{C}$ immediately, or processed for total RNA isolation directly.

Total RNA, genomic DNA isolation and gene cloning. Approximately $100 \mathrm{mg}$ samples of $S$. schenckii mycelia and yeast were separately pulverized under liquid nitrogen with a mortar and pestle. Total RNA isolation was carried out according to the manufacturer's protocol using the Trizol Reagent kit (Invitrogen, Carlsbad, CA, USA) and treated with the RNase-free DNase I kit from Takara Bio, Inc. (Tokyo, Japan) to eliminate DNA contamination. Genomic DNA was isolated from yeast phase colonies following the manufacturer's protocol using the InstaGene ${ }^{\mathrm{TM}}$ Matrix kit (Bio-Rad, Hercules, CA, USA). cDNA was synthesized from $500 \mu \mathrm{g}$ of total RNA of ATCC10268 by murine leukemia virus reverse transcriptase (MLV-RT) (Takara Bio, Inc.) primed with oligo(dT) following the manufacturer's instructions, and used as template for PCR. Degenerate primers, SsDRK1-F1 and SsDRK1-R1, were designed based on multiple alignments of the high conserved DRK1 domains of Coccidioides immitis (C. immitis) (EAS33695.2), Paracoccidioides brasiliensis (P. brasiliensis) (EEH34763.1), B. dermatitidis (EGE84246.1) and $H$. capsulatus $\mathrm{H} 88$ (EGC45940.1) amino acid sequences. PCR product of expected size was cloned into pMD18 vector (Takara Bio, Inc.) and sequenced. The degenerate primers yielded two fragments, with the length of 161 and $160 \mathrm{bp}$, respectively. Primers HBB-F and HBB-R were designed to amplify the cDNA sequence between the above two fragments. To obtain the full-length cDNA sequence of the SsDRKl gene, 5'-RACE and 3'-RACE were performed with 5'-Full RACE kit and 3'-Full RACE Core Set Ver.2.0 kit (Takara Bio, Inc.) according to the manufacturer's instructions. Nest-PCR was performed. Briefly, five specific primers CTE869-F and CTE869-R of 3'-RACE and R132-1, R132-2 and R132-3 of 5'-RACE were synthesized based on the cDNA sequence obtained by the degenerate primers. PCR products of 5'- and 3'-RACE were both cloned into pMD18 vector (Takara Bio, Inc.) and sequenced.

To determine the nucleotide sequence of the genomic DNA corresponding to the $S s D R K 1$, PCR was performed using the primers $S s D R K 1-P 1$ and SsDRK1-B3 and genomic DNA as template. The PCR products were then sequenced. The sequences of all the primers used in this study are listed in Table I.

Bioinformatics and phylogenetic analysis of SsDRK1. Nucleotide sequences and deduced amino acid sequences
Table I. Sequence of primers in this study.

\begin{tabular}{lc}
\hline Primer & Sequence (5'-3') \\
\hline DRK1-F1 & ACNGANAAYGTVAAYACYATGGC \\
DRK1-R1 & CGRTCMACCATRTBRTTGATNGT \\
HBB-F & TCACCAAAAAGATTGAGCGTCC \\
HBB-R & TGTCACCGTTGGCGATGGCTT \\
CTE869-F & GGCAACGCCATCAAGTTCACC \\
CTE869-R & GCTCGCGCTCACGGTTTTTTCGAGC \\
R132-1 (GSP1) & ATTCCCTTCACGCCCT \\
R132-2 (GSP2) & TGGTTTGTTGCAGTTGCAGGAT \\
R132-3 (GSP3) & TGAGATCACCGAACGCGACAGC \\
P1 & ATGACCGTTGTACCGACGAC \\
B3 & ATGTGAGGGCCTCTCTTAGC \\
$8 \mathrm{~F}$ & GAATCTGCACGGTATTCTGA \\
$58 \mathrm{R}$ & CTCAACCTCCACATCCTCAA \\
$24 \mathrm{~T}$ & FAM-CGTCGAGTCTGGTTACTAC-TAMRA \\
\hline
\end{tabular}

Degenerate primers designed based on multiple alignments of the high conserved DRK1 domains for gene cloning: DRK1-F1 and SsDRK1-R1. Primers designed to amplify the cDNA sequence: HBB-F and HBB-R. Primers of 3'-RACE: CTE869-F3, CTE869-F4, 5'-RACE: R132-1, R132-2 and R132-3. To determine the nucleotide sequence of the genomic DNA corresponding to the $S s D R K 1$, PCR was performed using the primers $P 1$ and $B 3$. Primers and a TaqMan probe of real-time RT-PCR: 8F, 58R and 24T.

of the cloned $S s D R K 1$ gene were analyzed. The nucleotide sequences were analyzed using Sequencer software (Sequencer, USA) and BLAST Network service of the National Center for Biotechnology Information (NCBI) (http://www.ncbi.nlm.nih. gov/blast). The open reading frame (ORF) was found by the ORF finder (http://www.ncbi.nlm.nih.gov/gorf/gorf.html). For the exact localization of the exon/intron boundaries the mRNAto-genomic alignment program Spidey (http://www.ncbi.nlm. nih.gov/IEB/Research/Ostell/Spidey/index.html) was used. The deduced amino acid sequence was analyzed with the Expert Protein Analysis System (http://www.expasy.org/) and the protein domain features of $S s D R K 1$ were determined by using Simple Modular Architecture Research Tool (http://hits. isb-sib.ch/cgi-bin/PFSCAN). Isoelectric point and molecular weight prediction were carried out at (http://cn.expasy.org/ tools/pi_tool.html). Multiple alignments of SsDRKl were performed with the ClustalW Multiple Alignment Program (http://www.ebi.ac.uk/clustalw/).

Differential expression of SsDRK1 in two stages during dimorphic switch. The expression of SsDRK1 transcript in different stages (mycelial, yeast) was measured by real-time RT-PCR. Primers and a TaqMan probe for target genes were designed with primer select in DNASTAR software (Lasergene) and are listed in Table I (24T, 8F, 58R). Fifty nanograms of total RNA were assayed from two stages of $S$. schenckii in triplicate using the PrimeScript RT-PCR kit (Takara Bio, Inc.). The minus-reverse transcriptase control was also performed in triplicate. The amplification conditions were optimized for the ABI PRISM-7500 instrument (Applied Biosystems). The cycling conditions using TaqMan probe detection were $95^{\circ} \mathrm{C}$ 


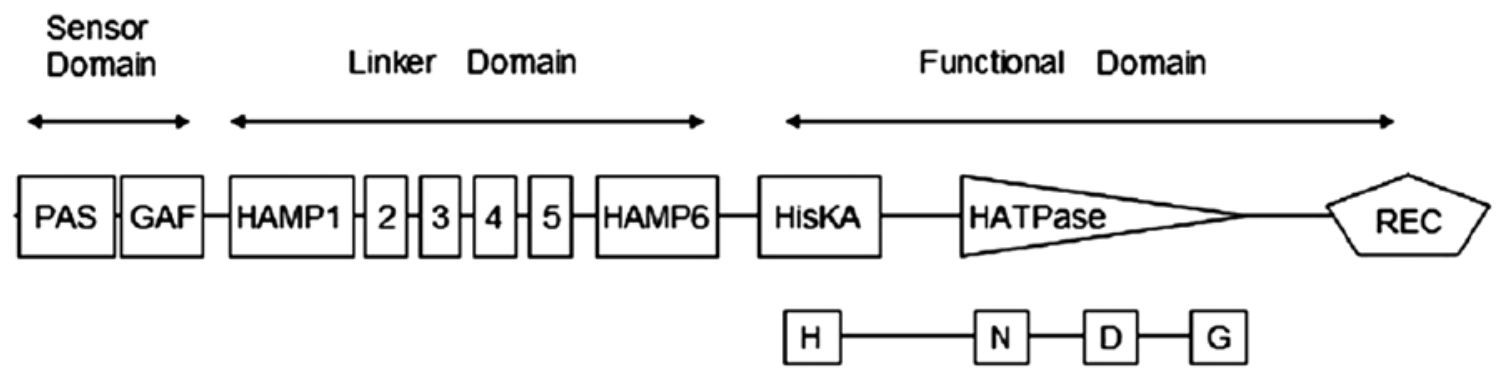

Figure 1. SSDRK1 domain organization: a schematic showing sensor, linker and functional domain organization, GAF and PAS motif located in the sensor domain, 6 HAMP are found at positions 231-746 in the linker domain, HisKA, HATPase and REC motif in context with H-, D-, G- and N-boxes were all identified in the functional domain.

Table II. Relative abundance of differential expression gene as determined by real-time RT/PCR $(\mathrm{mean} \pm \mathrm{SD})(\mathrm{P}<0.01)$.

\begin{tabular}{lccccc}
\hline cDNA name & Phase & Target $C_{\mathrm{T}}$ & 18 srDNA C & $\Delta \mathrm{C}_{\mathrm{T}}$ & $\Delta \Delta \mathrm{C}_{\mathrm{T}}$ \\
\hline DRK1 histidine kinase & Mycelial & $27.08 \pm 0.52$ & $20.66 \pm 0.27$ & $6.42 \pm 0.68$ & 0 \\
& Yeast & $23.90 \pm 0.26$ & $22.09 \pm 0.64$ & $1.81 \pm 0.87$ & $-4.61 \pm 0.23$ \\
\hline
\end{tabular}

$\Delta \mathrm{C}_{\mathrm{T}}=$ target transcript $\mathrm{C}_{\mathrm{T}}-18 \mathrm{srDNA} \mathrm{C}_{\mathrm{T}}$ normalization of $\mathrm{C}_{\mathrm{T}}$ for target gene relative to $18 \mathrm{srDNA} \mathrm{C}_{\mathrm{T}}$. Statistical analysis of normalized expression levels between mycelial and yeast form. Each of the target genes differs significantly (U-test, $\mathrm{P}<0.05) . \Delta \Delta \mathrm{C}_{\mathrm{T}}=\mathrm{mean}_{\mathrm{T}}$ yeast $\Delta \mathrm{C}_{\mathrm{T}}-\mathrm{mean}_{\mathrm{mycelial}} \Delta \mathrm{C}_{\mathrm{T}}$. The mean value for the mycelial $\Delta \mathrm{C}_{\mathrm{T}}$ was used as a calibrator to set the baseline for comparing mean differences in the $\Delta \mathrm{C}_{\mathrm{T}}$ values of the yeast form. 2- $\Delta \Delta \mathrm{C}_{\mathrm{T}}$, normalized target amount relative to the mycelial form.

for 2 min followed by 40 cycles at $95^{\circ} \mathrm{C}$ for $10 \mathrm{sec}, 61^{\circ} \mathrm{C}$ for $10 \mathrm{sec}, 72^{\circ} \mathrm{C}$ for $40 \mathrm{sec}$. 18srDNA was selected as the endogenous control. Relative quantification of target gene expression was evaluated using the comparative cycle threshold $\left(\mathrm{C}_{\mathrm{T}}\right)$ method as previously described by Livak and Schmittgen (5). The $\Delta \mathrm{C}_{\mathrm{T}}$ value was determined by subtracting the target $\mathrm{C}_{\mathrm{T}}$ of each sample from its respective $18 \mathrm{srDNA} \mathrm{C}_{\mathrm{T}}$ value. Calculation of $\Delta \Delta \mathrm{C}_{\mathrm{T}}$ involved using the mycelial sample $\Delta \mathrm{C}_{\mathrm{T}}$ value as an arbitrary constant to subtract from yeast sample $\Delta \mathrm{C}_{\mathrm{T}}$ values. Differences in expression of target genes were determined by $2^{-\Delta \Delta \mathrm{CT}}$. Data are expressed as arithmetic means \pm SD unless otherwise indicated. Comparison between mycelial and yeast samples was performed using the Student's t-test. Differences with a P-value of $<0.05$ were considered to be statistically significant.

\section{Results}

Cloning and genomic structure of SsDRK1. First, 828 bp cDNA fragment which had a high sequence similarity to the DRK1 of $P$. brasiliensis $\mathrm{Pb} 01$ was obtained from the total RNA of ATCC10268. Following RACE PCR, a fulllength SsDRK1 cDNA 4743 including an ORF of $4071 \mathrm{bp}$, encoding 1356 amino residues, was flanked by a 31 bp 5'-untranslated region (5'-UTR) and a 641 bp 3'-UTR. The most probable CAAT box is located at -2 , which is critical for eukaryotic transcription initiation (6). As in other PKC genes, no TATA box was identified within this sequence (7). Sequencing results showed that there is a poly (A) tails in 3'-UTR. The SsDRK1 genomic DNA is 4065 bp in length. The aligned results revealed that there are no introns between the sequences of the genomic DNA and the cDNA. Based on the sequence of cDNA, the molecular weight of the predicted amino acid is approximately $147.3 \mathrm{kDa}$, the theoretical $\mathrm{pI}$ is 5.46. Suggested models for transmembrane topology indicated that the amino acid sequence may be a soluble histidine kinase that lacks transmembrane segments. Fig. 1 shows that SsDRK1 contains three parts: sensor domain, linker domain and functional domain. The PAS and GAF domains, two structural families of cytoplasmic sensor domains, are found at positions 12-83 and 33-212 in the amino acid sequence. HAMP, which is an approximately 50 -amino acid $\alpha$-helical region, begins at position 231 in the linker domain part. It has been suggested that the HAMP domain possesses a role of regulating the phosphorylation of homodimeric receptors by transmitting the conformational changes in periplasmic ligand-binding domains to cytoplasmic signaling kinase domains. The functional domain of $S S D R K 1$ is predicted to have the necessary elements for histidine kinase function, including the histidinecontaining H-box and aspartate containing D-box involved in phosphorelay. The sequence also contains the $\mathrm{N}$-and G-boxes used in ATP-binding and catalytic function, and an aspartatecontaining receiver domain (Fig. 4). $S s D R K 1$ is homologous to the hybrid histidine kinase SLN1 in Saccharomyces cerevisiae (S. cerevisiae), DRK1 in B. dermatitidis and to sequences in the genomes of $H$. capsulatum and $C$. immitis, dimorphic fungi for which extensive genome sequence is available.

Homology and phylogenetic analysis of SsDRK1. Multialignment analysis by ClustalW indicated that $S s D R K 1$ has a high identity to DRK1 reported in other species, sharing a similarity of $66 \%$ identity to P. brasiliensis (EEH34763.1), $65 \%$ identity to $B$. dermatitidis (EGE84246.1), 65\% identity to $C$. immitis (EAS33695.2), 67\% identity to $H$. capsulatus (EGC45940.1) (Fig. 2). Based on the results of the alignment of DRK1 sequences of the former and some common 

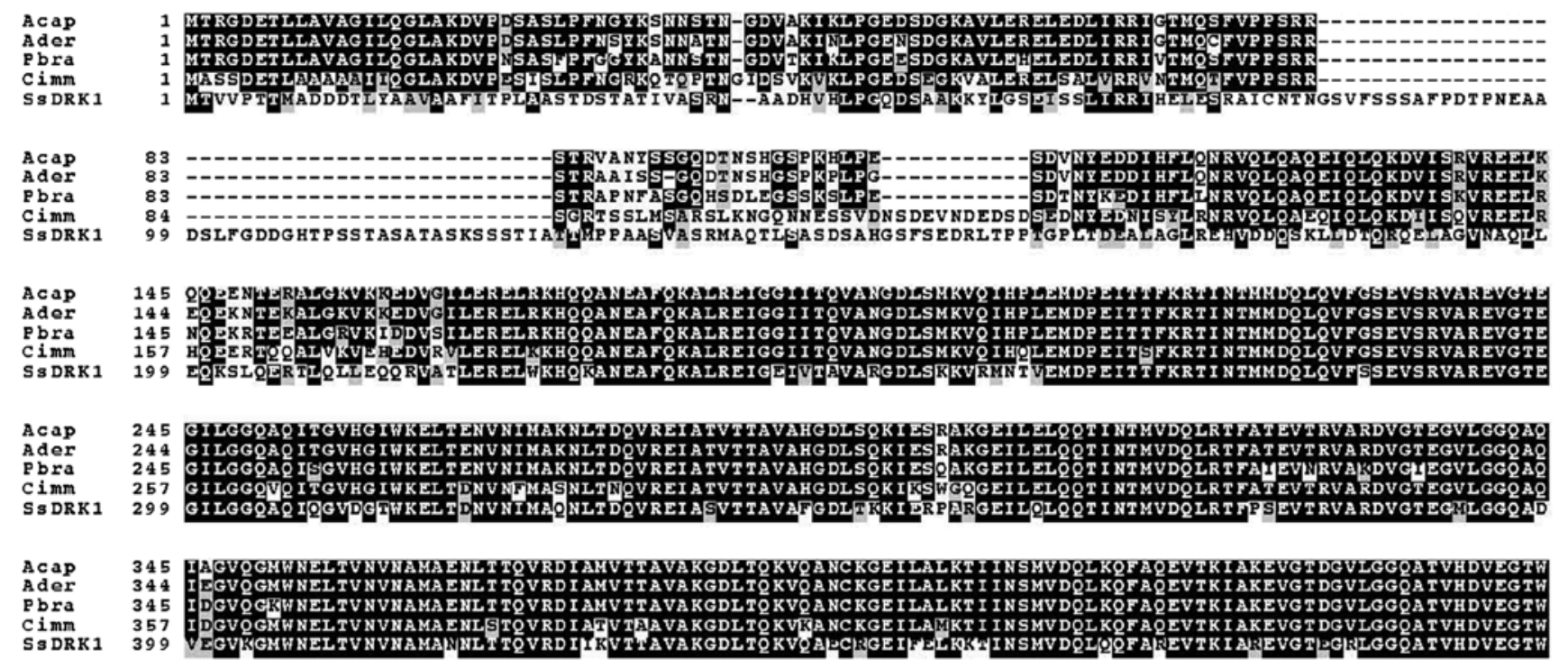

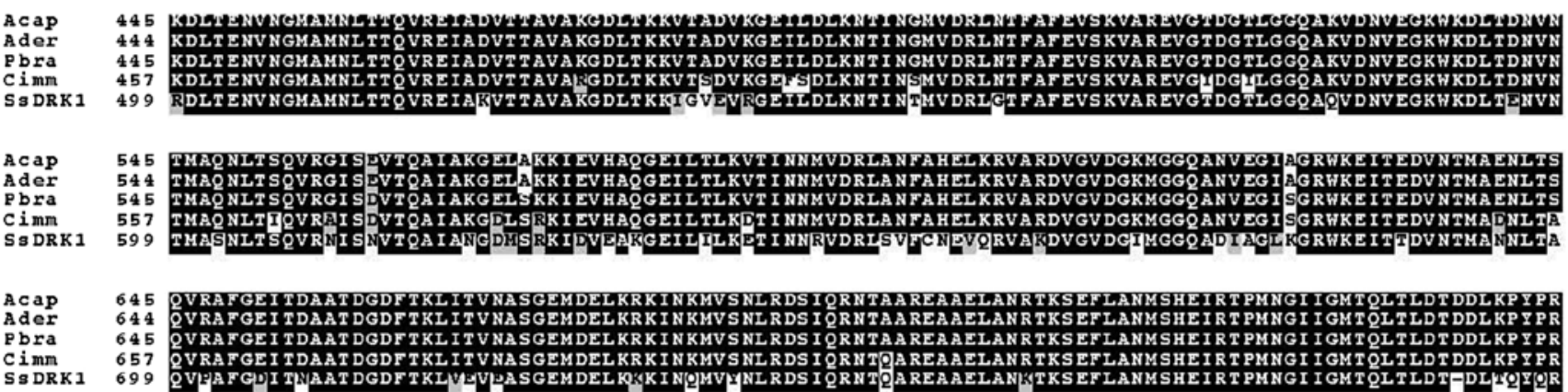

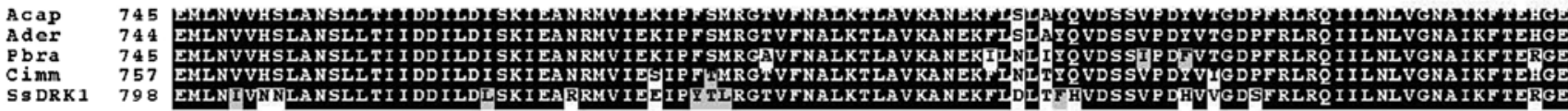

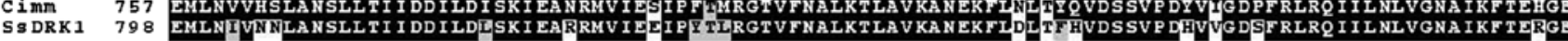

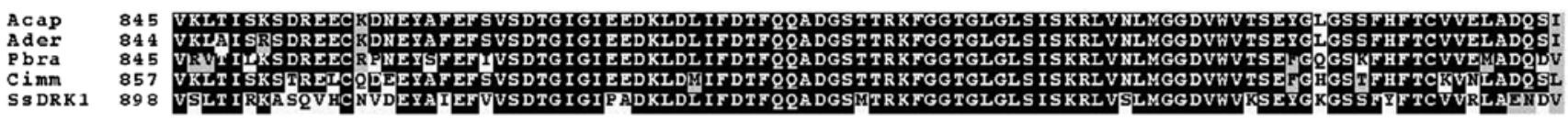

ACaP 945 RMISASLLPYKMHRVLF IDKG TGGHAEETTEMLKOLDLEPLVVKDESOVPPPEIQDPTGKDSGHAY DVI IVDSVDTAQ NLRT YDEFKY IP IVLLCPVVS

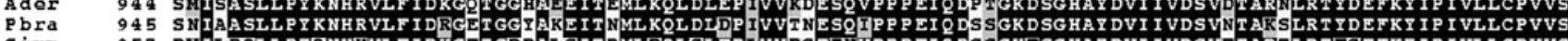

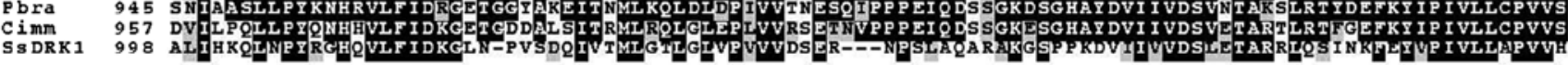

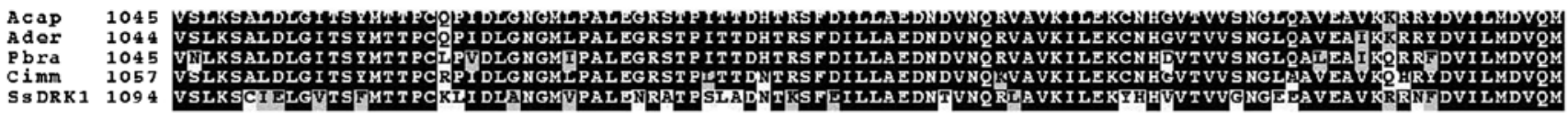

AC AP 1145 PVHGGFATGKIREYEKSEG-LSRTP I TALTAHAMLGDREKCIOAQMDLYLAKPLKONOMIOTILKCATLGGSLIEKSKEPRMSS SGEP HHHVP NSNGLK Ader 1144 PVMGGFEATGKIREYEKKNG-LSRTP I ALTA HAMLGDREKCIOAQMDEYLAKPLKONOMIOTILKCATLGGSLLEKSKEPRMSS SGEP PHHVP SNGMK

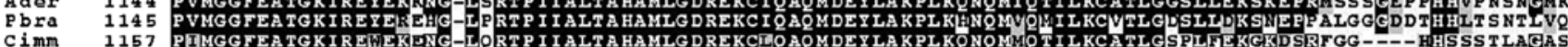

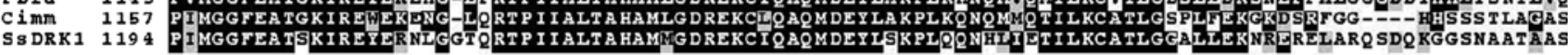

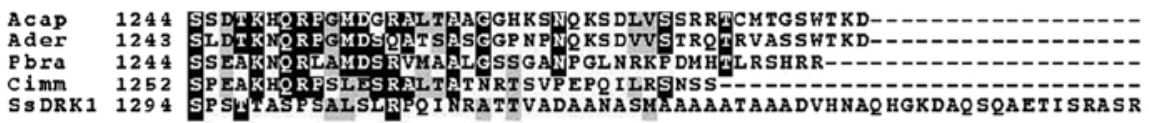

Figure 2. Multiple sequence alignment of $S s D R K 1$ to other fungal HK homologs. Amino acid sequence alignment of $S s D R K 1$ with other HK homologs is shown. The amino acid sequence of Ajellomyces capsulatus (Histoplasma capsulatus), Ajellomyces dermatitidis (Blastomycosis dermatitidis), Paracoccidioides brasiliensis and Coccidioides immitis were aligned using the online version of ClustalW. Shade residues indicate $\geq 75 \%$ homology (black) or $\geq 50 \%$ homology (gray).

fungi, the phylogenetic trees were constructed using the ClustalW software (Fig. 3). Three groups were clearly generated in the phylogenetic tree. The SsDRKI identified in this study appeared most closely related to sequences from Neurospora crassa (N. crassa), a member of the ascomycetous class pyrenomycetes. The results also suggested that the evolu- 


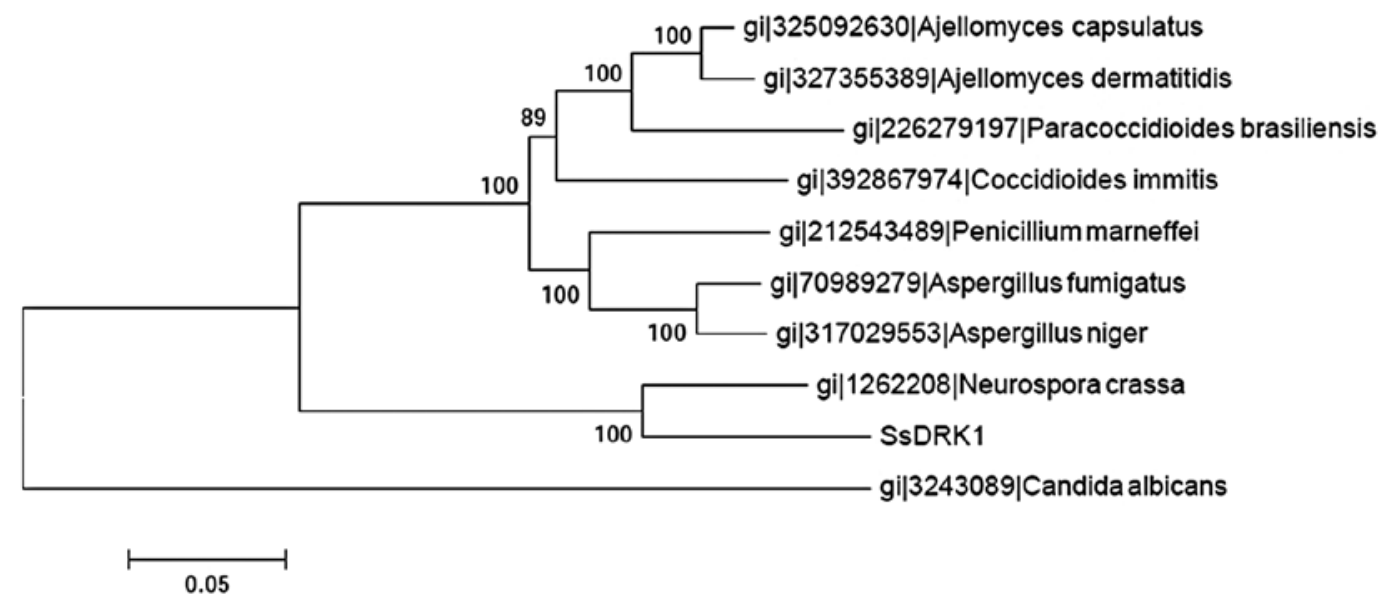

Figure 3. Phylogenetic relationship among DRK1 homologs. An NJ tree was generated by MEGA version 5.05 with bootstrap analysis based on 500 replications. Percentage bootstrap values are shown at branch points. Scale bar indicates the number of substitutions per site.

\section{G Box \\ Sc GTGLGLSICRQLANMWHGTIKLESKVGVGSKFTFLPL \\ Bd GTGLGLSISKRLVNLMGGDVWVTSEYGLGSSFHFTCV \\ Hc GTGLGLSISKRLVNLMGGDVWVTSEYGLGSSFHFTCV \\ Ci GTGLGLSISKRLVNLMGGDVWVSTEFGHGSTFHFTCK \\ Ss GTGLGLSISKRLVSLMGGDVWVKSEYGKGSSFYFTCV}

Figure 4. SsDRK1 has the domain structure and sequence of histidine kinase and is conserved in dimorphic fungi. SsDRK1 has a histidine-containing H-box, an aspartate-containing D-box, and G- and N-boxes (7). Sequences homologous to the S. cerevisiae (Sc) histidine kinase SLN1 and B. dermatitidis (Bd) histidine kinase are present in other dimorphic fungi H. capsulatum $(\mathrm{Hc})$ and C. immitis $(\mathrm{Ci})$.

tionary relationship of $S s D R K 1$ might be different from that in Candida albicans (C. albicans).

Expression of SsDRK1 in two stages of Sporothrix schenckii. The mRNA expression of SsDRK1 in different stages was analyzed by real-time RT-PCR normalized against 18SrDNA levels. Following amplification, $\mathrm{Ct}, \Delta \mathrm{Ct}$ and $\Delta \Delta \mathrm{Ct}$ values were calculated. Expression was determined as fold increased $2^{-\Delta \Delta C t}$ levels relative to the stage with lowest expression (mycelia) set to 1 . The SsDRK1 gene was expressed in two stages of $S$. schenckii, with higher mRNA levels observed in yeast (24.42-fold). There were significant differences between the mycelial and the yeast form (Table II).

Accession number. The full length of cDNA sequence and genomic DNA sequence of the $S s D R K 1$ gene were submitted to the GenBank database under the accession number JX312331 and JX416706, respectively.

\section{Discussion}

Histidine protein kinases (HPKs) are a large family signaltransduction enzymes that autophosphorylate on a conserved histidine residue. HPKs form two-component signaling systems together with their downstream target proteins, the response regulators, which have a conserved aspartate in a 'receiver domain' that is phosphorylated by the HPK. The dimorphism regulating kinase DRK1 was recently proved to mediate the thermally induced transition to the pathogenic yeast-phase program in both $B$. dermatitidis and $H$. capsulatum (3). In this study, based on the conserved structures of the DRK1 in four types of fungi cells, the degenerate primers were designed to obtain the homologs of DRK1 in S. schenckii. The production of PCR has a very high identity to the DRK1 of $P$. brasiliensis $\mathrm{Pb} 01$. The ORF of $S s D R K 1$ encoded protein was mostly similar in identity to the DRK1 of $N$. crassa, similar with previous molecular phylogenetic analyses both 
on a pertussis toxin-sensitive $G$ protein $\alpha$ subunit (8) and three chitin synthase genes (9). Aligned to the other fungal DRK1, the identities were 64 to $74 \%$. However, SsDRK1 shares limited sequence similarity with histidine kinases that regulate filamentation in the more distantly related fungus C. albicans.

The amino sequence of $S s D R K 1$ is predicted to have the necessary elements for histidine kinase function including H-box, D-box, N- and G-boxes. This indicates that the SsDRKI has similar functions to other fungi histidine kinases. The typical HPK is a transmembrane receptor with an aminoterminal extracellular sensing domain and a carboxy-terminal cytosolic signaling domain; however, a type of soluble histidine kinase that lacks transmembrane segments was also identified. The cytoplasmic sensor domain including GAF, PAS and PCD may reside $\mathrm{N}$-terminal to the $\mathrm{C}$-terminal transmitter domain in the soluble histidine kinase (10). SsDRK1 in the present study was proved to be lacking transmembrane segments and carrying GAF and PAS domains in the sensor part, which suggested that $S s D R K 1$ is a soluble histidine kinase.

Histidine kinase two component signaling systems have recently been shown to play the role in environmental sensing and all development in eukaryotes. In C.albicans, they regulate filamentation whereas in B. dermatitidis and $H$. capsulatum, they may control phase transition and virulence gene expression as well as cell development and sporulation in the other systemic dimorphic fungi. Does $S s D R K 1$ have the same functions during the process of dimorphic switch in S. schenckii? In this study, the mRNA expression of SsDRK1 in yeast cells was higher than in mycelial cells, which suggested that $S s D R K 1$ is involved in regulating phase transition.

What is the environmental signal that $S s D R K 1$ senses to regulate phase transition and virulence gene expression? In $S$. cerevisiae (11), histidine kinase $S \ln 1 p$ detects osmotic stress, whereas in Schizosaccharomyces pombe (12), the histidine kinase-regulated SPC1 MAPK cascade senses osmotic, oxidative, heat stress and nutrient deprivation. Potential signals for histidine kinase sensing in dimorphic fungi include temperature, osmotic or oxidative stress, nutrient deprivation, redox potential, and host-derived factors including hormones such as 17- $\beta$-estradiol, which induces germ tubes in $C$. albicans (13) and block mold-to-yeast transition of P. brasiliensis (14). In this study, the mycelial cells of S. schenckii switched to yeast cells when they were incubated in BHI liquid medium at $37^{\circ} \mathrm{C}$, which suggests $S s D R K 1$ can detect the change of temperature and nutrient deprivation in the environment.

The detailed functions of the SsDRK1 and its up- and down-stream proteins as well as their interactions require further investigation. If the formation mechanism of the yeast cells (the parasitic form) of $S$. schenckii is elucidated, this may lead to a therapy strategy for sporotrichosis.

\section{Acknowledgements}

This study was partly supported by a grant from the National Natural Science Foundation of China (grant no. 81000069). The authors thank Zhang Zhenying and Yu Zhen for their assistance with image and statistical analysis.

\section{References}

1. Travassos LR and Lloyd KO: Sporothrix schenckii and related species of Ceratocystis. Microbiol Rev 44: 683-721, 1980.

2. Guarro J, Gené J and Stchigel AM: Developments in fungal taxonomy. Clin Microbiol Rev 12: 454-500, 1999.

3. Nemecek JC, Wuthrich M and Klein BS: Global control of dimorphism and virulence in fungi. Science 312: 583-588, 2006.

4. Zhang ZY, Hou BB, Xin Y and Liu XM: Protein profiling of the dimorphic pathogenic fungus, Sporothrix schenckii. Mycopathologia 173: 1-11, 2012.

5. Livak KJ and Schmittgen TD: Analysis of relative gene expression data using real-time quantitative PCR and the 2(-Delta Delta C(T)) method. Methods 25: 402-408, 2001.

6. Feng P, Xie Z, Sun J, Zhang J, Li X and Lu C and Xi L: Molecular cloning, characterization and expression of PmRsrl, a Ras-related gene from yeast form of Penicillium marneffei. Mol Biol Rep 37: 3533-3540, 2010.

7. Aquino-Piñero E and Rodríguez-del Valle N: Characterization of a protein kinase $\mathrm{C}$ gene in Sporothrix schenckii and its expression during the yeast-to-mycelium transition. Med Mycol 40: 185-199, 2002.

8. Delgado N and Rodríguez-del VN: Presence of a pertussis toxinsensitive G protein alpha subunit in Sporothrix schenckii. Med Mycol 38: 109-121, 2000.

9. Chua SS, Momany M, Mendoza L and Szaniszlo PJ: Identification of three chitin synthase genes in the dimorphic fungal pathogen Sporothrix schenckii. Curr Microbiol 29: 151-156, 1994.

10. Kimura S, Shiraiwa Y and Suzuki I: Function of the N-terminal region of the phosphate-sensing histidine kinase, $\mathrm{SphS}$, in Synechocystis sp. PCC 6803. Microbiology 155: 2256-2564, 2009.

11. Van Wuytswinkel O, Reiser V, Siderius M, Kelders MC, Ammerer G, Ruis H and Mager WH: Response of Saccharomyces cerevisiae to severe osmotic stress: evidence for a novel activation mechanism of the HOG MAP kinase pathway. Mol Microbiol 37: 382-397, 2000.

12. Pöhlmann J and Fleig U: Asp1, a conserved 1/3 inositol polyphosphate kinase, regulates the dimorphic switch in Schizosaccharomyces pombe. Mol Cell Biol 30: 4535-4547, 2010.

13. Cheng G, Yeater KM and Hoyer LL: Cellular and molecular biology of Candida albicans estrogen response. Eukaryot Cell 5: 180-191, 2006.

14. Salazar ME, Restrepo A and Stevens DA: Inhibition by estrogens of conidium-to-yeast conversion in the fungus Paracoccidioides brasiliensis. Infect Immun 56: 711-713, 1988. 